\title{
Joint action in a cooperative precision task: nested processes of intrapersonal and interpersonal coordination
}

\author{
Verónica C. Ramenzoni · Tehran J. Davis • \\ Michael A. Riley $\cdot$ Kevin Shockley • \\ Aimee A. Baker
}

Received: 18 November 2010/ Accepted: 22 March 2011/Published online: 9 April 2011

(C) Springer-Verlag 2011

\begin{abstract}
The authors determined the effects of changes in task demands on interpersonal and intrapersonal coordination. Participants performed a joint task in which one participant held a stick to which a circle was attached at the top (holding role), while the other held a pointer through the circle without touching its borders (pointing role). Experiment 1 investigated whether interpersonal and intrapersonal coordination varied depending on task difficulty. Results showed that interpersonal and intrapersonal coordination increased in degree and stability with increments in task difficulty. Experiment 2 explored the effects of individual constraints by increasing the balance demands of the task (one or both members of the pair stood in a less stable tandem stance). Results showed that interpersonal coordination increased in degree and stability as joint task demands increased and that coupling strength varied depending on joint and individual task constraints. In all, results suggest that interpersonal and intrapersonal coordination are affected by the nature of the task performed and the constraints it places on joint and individual performance.
\end{abstract}

Keywords Interpersonal coordination · Joint action · Postural control

\footnotetext{
V. C. Ramenzoni · T. J. Davis · M. A. Riley · K. Shockley

A. A. Baker

Department of Psychology,

University of Cincinnati, Cincinnati, OH, USA

V. C. Ramenzoni $(\bowtie)$

Communication before Language, Max Planck Institute,

Wundtlaan 1, 6525 Nijmegen, The Netherlands

e-mail: veronica.ramenzoni@mpi.nl
}

\section{Introduction}

Human interactions are governed by principles. Some of these principles have to do with social conventions (e.g., turn-taking during conversations), and others with the neural, cognitive, and perceptual-motor processes that support interactions. Joint tasks (e.g., joint attention, communication, task sharing, and team sports) require two or more agents to coordinate-intentionally or spontaneously - to attain a common goal (e.g., Richardson and Dale 2005; Richardson et al. 2007a; Sebanz et al. 2006). In dyadic joint tasks, for instance, the actions of one agent are linked to those of the other, and successful performance often depends on each agent's ability to detect and respond to the other's behavior. This ability to engage in and sustain reciprocal relations is regulated by cognitive (Wilson and Knoblich 2005) and perceptual-motor processes (Shockley et al. 2003; Stoffregen et al. 2009), which work in concert to enable coordination during joint action (Sebanz et al. 2006). In this study, we investigated the perceptual-motor processes that sustain the performance of a dyadic joint task. We evaluated parallel changes in coordinative dynamics within each individual and between individuals when changes in dyadic (Experiments 1 and 2) and individual (Experiment 2) task constraints were introduced.

Interpersonal and intrapersonal coordination

The contribution of the perceptual-motor system to dyadic interactions is twofold: It modulates the movements of the agent's body with respect to the movements of the other agent (i.e., interpersonal coordination) and simultaneously controls and coordinates the movements of the agent's own individual body segments (i.e., intrapersonal 
coordination), for example to maintain postural stability during the interaction. Differences and similarities in these two scales of coordination have been extensively studied (Amazeen et al. 1995; Oullier et al. 2008; Schmidt et al. 1990, 1998; Schmidt and Turvey 1994; Temprado et al. 2003), primarily in the context of rhythmic bimanual coordination tasks that can be performed by an individual who uses both hands, or by a dyad wherein each person uses one hand. Studies that directly compared intrapersonal and interpersonal rhythmic bimanual coordination have shown that coupling strength is weaker for interpersonal than intrapersonal coordination, but nevertheless the coordinative processes share striking similarities across scales. Both types of coordination evidence comparable transitions between stable coordination patterns and are similarly affected by changes in movement frequency and frequency detuning. The same basic dynamical mechanisms seem to underlie intrapersonal and interpersonal coordination.

The dynamical mechanisms of coordinative processes are encapsulated in the idea of coordinative structures or synergies - temporary assemblies of neuromuscular elements that function as a collective unit. The formation of synergies effectively reduces the degrees of freedom controlled directly by the CNS. Synergies exploit neuromotor redundancies to provide multiple, equivalent motor solutions while also providing stability via reciprocal compensations for unwanted perturbations and fluctuations (Bernstein 1967; Gelfand and Tsetlin 1966; Latash 2008; Turvey 1977, 1990; Turvey et al. 1978; Whiting et al. 1992). It has been proposed that synergies can exist at the interpersonal scale as well as at the scale of an individual actor's neuromotor system (Black et al. 2007; Fowler et al. 2008; Latash 2008; Marsh et al. 2006, 2009; Richardson et al. 2010; Schmidt and Richardson 2008; Richardson et al. 2008; Riley et al. 2011; Shockley et al. 2009). This project investigates how synergies at the interpersonal and intrapersonal scales respond to changes in task constraints in the context of a joint supra-postural task.

Supra-postural tasks are behaviors that people perform while standing, but that have goals that differ from the control of stance (Riccio and Stoffregen 1988; Riley et al. 1999; Stoffregen et al. 1999, 2000). For example, people often stand while performing manual tasks that require precision, such as aiming at a target (Balasubramaniam et al. 2000). Postural control and supra-postural manual control must be integrated in some sense in order to satisfy the performance demands of a manual task like aiming, because excessive body sway might make it difficult to hit the target. The task used in the current study required one actor to hold a pointer inside a target ring held by another actor, requiring coordination at the intrapersonal and interpersonal scales. At the intrapersonal scale, the demands of the precision manual task must be integrated with postural control. This might require a reduction in postural sway variability or coordination of hand and torso motion, for example, to prevent postural sway from giving rise to unwanted hand movements. At the interpersonal scale, the task requires the pointer and the holder to control their respective movements with respect to how the other person is moving. This minimally might require interpersonal coordination of hand movements, but might also be associated with interpersonal postural coordination.

The demands of this joint supra-postural task can be manipulated at both the individual (intrapersonal) and the joint (interpersonal) scales. For example, at the intrapersonal scale having participants stand in an unstable posture can challenge balance control. Likewise, at the interpersonal scale, the difficulty of the task can be manipulated directly by varying the size of the target. When this joint task requires less precision (i.e., the targets are larger), the coordination between actors decreases (Ramenzoni et al. under review). In the present study, we employed manipulations that targeted both the individual and the joint scales in order to address the question of how constraints operating simultaneously at those scales influence intrapersonal and interpersonal coordinative processes involved in joint task performance. In Experiment 1, we manipulated the difficulty of the task by varying the target size. In Experiment 2, we added a manipulation at the individual scale by requiring one participant to adopt a tandem Romberg (heel-to-toe) stance, which destabilizes balance and increases medio-lateral (ML) postural sway.

Broadly speaking, we expected challenges to task performance to be countered by specific changes in intrapersonal and interpersonal coordination, thereby revealing some of the perceptual-motor principles that govern interactions during joint task performance. In Experiment 1, we expected that increasing task difficulty at the joint scale would increase intrapersonal (i.e., hand-torso) and interpersonal coordination (especially of the participants' hands). Although distinct mechanisms may instantiate coordinative relations at those two scales, parallel effects would indicate interdependence across scales. Interdependence could arise because intrapersonal coordination is nested within a more global process of interpersonal coordination, and coordination at both scales is instantiated in overlapping structures of the same system. In Experiment 2, we simultaneously manipulated task demands at the joint and individual scales to further probe the issue of relative interdependence versus independence across scales of coordination. Increasing the task demands at the individual scale could introduce either perturbations or compensatory changes in the stability of coupling relations at one or both scales. If manipulations introduced at a given scale affected only that scale, the results would suggest 
independence of processes of intrapersonal and interpersonal coordination rather than interdependence (see introduction to Experiment 2 for a detailed explanation of alternatives that would lead to distinct patterns of results).

\section{Experiment 1}

Experiment 1 determined whether variation in joint task demands would induce changes in interpersonal and intrapersonal synergies. Participants' hand and torso movements were recorded while they worked together to perform the supra-postural joint aiming task. One participant had to keep a pointer within the boundaries of and without touching the target circle, which was held by the other participant. The motor requirements of the two roles (i.e., pointing and holding) were essentially identical (standing with one arm extended in a stable, shoulderwidth stance, on a firm surface; see Fig. 1). Participants were not explicitly asked to coordinate their hand or torso movements; however, given the nature of the task, a lack of coordination (at least of hand movements) was likely to result in poor performance.

Joint task difficulty was manipulated by varying the size of the circle; the smaller the circle, the harder the task. Based on findings on individual supra-postural task performance (Balasubramaniam et al. 2000), significant changes in motion dynamics were expected for the direction of motion more directly targeted by the task-mediolateral (ML) motion-so we report only ML data. More demanding joint task difficulty conditions were expected to

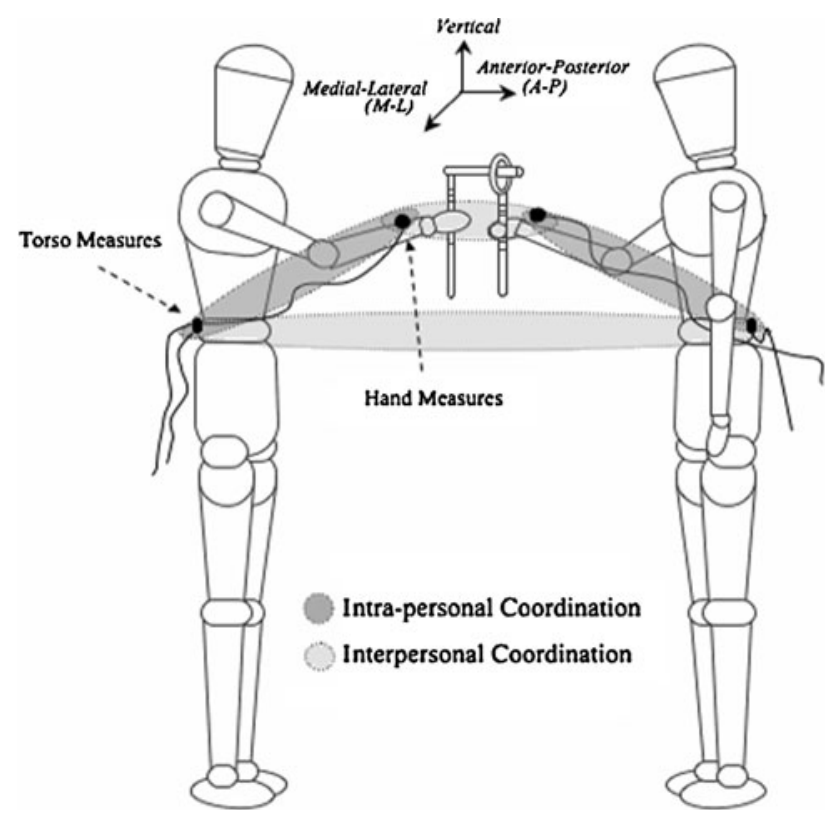

Fig. 1 Depiction of the task and experimental setup result in enhanced coordination at the intrapersonal scale (i.e., hand-torso coordination). Such enhancements of intrapersonal coordination would allow, for instance, torso motion to be compensated by hand movements-a reflection of the reciprocal coupling that is characteristic of synergies (Latash 2008). Interpersonal coordination (i.e., hand-hand and torso-torso coordination) was also expected to increase in the more difficult condition, as individuals are required to monitor each other's movements more closely and respond to the other person's movements so as not to touch the borders of the smallest circle. Because hand movements are more directly relevant to task performance, this effect was expected to be more salient for the coordination between hands than torsos.

Coordination at the intrapersonal (i.e., hand-torso coordination for individual participants) and interpersonal (hand-hand or torso-torso coordination between individuals) scales was quantified using cross-recurrence quantification analysis (CRQA; Shockley 2005; Shockley et al. 2002; Zbilut and Webber 1992). Changes in coordinative dynamics and the mechanism(s) underlying them can be indexed using two CRQA measures. The degree of coordination is measured by the quantity \%Recurrence (basically the overall degree of overlap of the two trajectories-the extent to which segments shared similar configurations during the trial) and the strength of coupling (i.e., stability of coordinative relations) by the quantity MaxLine (the maximum amount of time the two time series maintain common trajectories). Functional changes in coordination, such as the ones predicted here, could be attained via distinct mechanisms indicated by these two measures. Coordination could be enhanced by reducing the magnitude of noise in the motor system (reflected by an increase in \%Recurrence) and/or by increasing coupling strength (reflected by an increase in MaxLine) (Pellecchia et al. 2005; Richardson et al. 2007a; Shockley and Turvey 2005, 2006). Because these mechanisms can lead together or separately to improvements in coordination, here we report results for \%Recurrence and MaxLine for intrapersonal and interpersonal coordination.

Methods

\section{Participants}

In both experiments, procedures were approved by the IRB and all participants gave informed consent before participating. Twenty-four participants (12 women, 12 men; mean age $=19.59$ years) from the University of Cincinnati participated in Experiment 1. Pairs of participants were of the same gender and differed in height by $1-5 \mathrm{~cm}$ $($ mean $=2.38 \mathrm{~cm} ; \mathrm{SD}=1.67)$ and in shoulder-to-fingertip length by $0.5-9.5 \mathrm{~cm}($ mean $=0.86 \mathrm{~cm} ; \mathrm{SD}=2.59 \mathrm{~cm})$. 
All participants were right-handed and had normal or corrected-to-normal vision.

\section{Materials and apparatus}

Participants' right hand and torso movements in the ML plane were recorded at a rate of $60 \mathrm{~Hz}$ using a Polhemus Fastrak electromagnetic motion capture device (Polhemus Corporation, Colchester, VT) with 6D Research System data collection software (Skill Technologies, Inc., Phoenix, Arizona) running on a PC. The motion tracker sensor for the torso was attached by a Velcro belt that held the sensor on the small of the back, at approximately the 4th lumbar vertebra. Adhesive tape was used to secure the hand sensor on the back of the hand.

The supra-postural precision task was performed using a pointer and target circles, each constructed using $30-\mathrm{cm}-$ long wooden handles with additional materials attached at the top. Attached perpendicular to the handle of the pointer was a second wooden stick that was $16 \mathrm{~cm}$ long with a diameter of $1.25 \mathrm{~cm}$; this piece defined this object as the pointer, and it was to be held within the circle. The target circles were plastic rings of varying diameters $(10,6$, and $2.5 \mathrm{~cm}$ ) attached to the handle (see Fig. 1).

\section{Procedure}

Before the beginning of the experiment, participants' anthropometric measures were recorded. Afterward, pairs of participants were asked to perform a precision task with three difficulty levels. The three difficulty conditions corresponded to changes in the size of the target circle, with the easiest being the largest circle and the hardest the smallest circle. The role performed in the task (holder or pointer) was counterbalanced across participants, so that each participant performed both roles for each difficulty condition. Difficulty conditions were presented in random order. Each participant completed a total of 12 trials, with one trial per condition. Each trial lasted $50 \mathrm{~s}$.

Throughout the experiment, participants stood barefoot with their feet shoulder width apart. Participants stood at a fixed location that was marked on the floor and faced each other. The distance between the participants was determined as the distance at which they could touch fingertips with the right arm in extension, $-5 \mathrm{~cm}$.

Instructions were given to pairs of participants together. Pairs were instructed to perform the task by maintaining the pointer inside in the circle without touching the borders for the duration of each trial. Participants were asked not to lock their knee joints, to keep their right elbow flexed at approximately a $135^{\circ}$ angle and relaxed (this arm posture was demonstrated to them by the experimenter), and to try to avoid gross and sudden body movements (such as shaking the head or the arm). Participants were also instructed not to talk during trials and to focus their eyes on the task (i.e., look at the pointer and circle). Trials in which participants failed to comply with task demands (i.e., the pointer touched the circle at least once) were repeated at the end of the experimental session. Halfway through data collection pairs were given a mandatory 5-min break to minimize the effect of fatigue on performance.

\section{Data reduction and analysis}

Measures of interpersonal (hand-hand and torso-torso coupling) and intrapersonal (hand-torso coupling) coordination were computed from the recorded ML hand and torso trajectories using CRQA (Zbilut et al. 1998). For interpersonal coordination, separate CRQA analyses were performed on the hand and the torso data, which yielded measures of hand-hand coordination and torso-torso coordination across participants. For intrapersonal coordination, CRQA was performed across hand and torso data yielding measures of hand-torso coordination for each member of the pair. Motion variability for the each participant's hands and torsos was quantified by calculating the overall standard deviation of each segment in the ML plane of motion for each trial. Values of these measures for each condition (CRQA measures and SDs) were then analyzed using ANOVA with task difficulty (easiest, middle, and hardest) as factors, ${ }^{1}$ followed by BonferroniDunn post hoc tests. Only significant results are reported.

\section{Results and discussion}

Overall, participants succeeded in performing the task; trials were repeated only in two occasions for one pair due to the pointer touching the circle.

Interpersonal coordination (as measured by \%Recurrence) was affected by changes in task difficulty. The movements of the hands $[F(2,11)=8.57, P<.05$, $\left.\eta_{p}^{2}=0.44\right]$ and torsos $\left[F(2,11)=3.99, P<.05, \eta_{p}^{2}=0.27\right]$ became increasingly coordinated across members of the pair as joint task difficulty increased. However, no differences in interpersonal coordination stability (MaxLine) were found. The pattern of outcomes of post hoc tests subsequent to the task difficulty main effect for \%Recurrence showed that hand-hand coordination exhibited slightly greater sensitivity to the varying task-difficulty constraints than torso-torso coordination. Hand-hand coordination exhibited a significantly higher percentage of

\footnotetext{
${ }^{1}$ All observations in the ANOVA on individual performance (intrapersonal CRQA and SD measures) were not independent given that members of a pair coordinated their movement in order to perform the joint precision task.
} 
shared configurations for the hardest compared with both the easiest and the middle difficulty conditions (easier: $m=8.5, \mathrm{SD}=1.9$; middle: $m=10.7, \mathrm{SD}=3.5$; hardest: $m=13.7, \mathrm{SD}=4.8$ ) (see Fig. 2, right panel). Torso-torso coordination, however, only showed a significant increase for the hardest compared with the easiest difficulty condition (easier: $m=5.5, \quad \mathrm{SD}=1.3$; middle: $m=6.1$, $\mathrm{SD}=1.3$; hardest: $m=7.7, \mathrm{SD}=1.6$ ) (see Fig. 2, middle panel).

Increases in coordination at the interpersonal scale were accompanied by an increase in hand-torso coordination at the intrapersonal scale as joint task difficulty increased. Intrapersonal coordination (as measured by \%Recurrence) increased significantly for the hardest task difficulty condition compared with both the easiest and the middle difficulty conditions, $F(2,46)=9.41, \quad P<.05, \quad \eta_{p}^{2}=0.29$, (easier: $m=7.7, \mathrm{SD}=3.3$; middle: $m=7.8, \mathrm{SD}=2.8$; hardest: $m=9.8, \mathrm{SD}=3.5$ ) (see Fig. 2, left panel). These results suggest that, to an extent, intrapersonal coordinative structures adjusted to meet specific task demands, while simultaneously permitting or sustaining coordinative processes at the interpersonal scale.

This pattern of enhanced coordination at both scales emerged against a backdrop of increased movement variability (which was measured by the SD of the segment trajectories). Though the joint task difficulty manipulation did not affect hand motion, participants' torso motion was more variable for the difficult (small circle) compared with the easy and middle conditions, $F(2,46)=16.40, P<.05$, $\eta_{p}^{2}=0.42$. It is important to note that the measure SD merely indexes the amount of variability of the segments' trajectories, irrespective of the source of that variability (e.g., random neuromotor noise might increase variability, but so it might a completely deterministic factor). This finding can therefore be reconciled with a finding of greater $\%$ Recurrence (reduced noise magnitude) between participants' torsos. Torso motion is one way of measuring postural sway and as such provides a summary measurement of the impact of external and internal forces on the body and the body's actions to preserve balance. During single- person precision tasks, postural sway becomes less variable as the precision demands of the task increase, reflecting an increment in postural stability (Balasubramaniam et al. 2000). The current findings indicate that this is not necessarily the case for dynamic joint tasks, wherein one person has to move to track the movements of the other. A likely explanation for the observed increments in torso variability is that it might indicate an adaptive strategy deployed by each member of the dyad, thus reflecting the need for participants to move more in order to closely counter or track the movements of the other member of the dyad-in effect, one person's movements caused subsequent movements by the other person, which then amplified the other person's movements further, and so on.

For both interpersonal and intrapersonal coordination, increases in task difficulty were associated with increased $\%$ Recurrence, reflecting a greater tendency for the body segments to share common configurations at both scales of coordination, which may translate to a reduced amount of noise in the coordination pattern. The finding that there was no corresponding influence of task difficulty on MaxLine indicates that although there were more shared configurations when the task was more difficult (suggesting that hand and torso movements were more tightly constrained), their coordination was no more stable (i.e., shared trajectories did not persist any longer than in the easier task condition).

Increasing the difficulty of the task - a manipulation that targeted the scale of the joint task, since task difficulty (at least defined operationally in terms of target size) has no meaning at the individual scale-increased the degree of both interpersonal and intrapersonal coordination. Moreover, enhancement of intrapersonal coordination occurred against a background of increased variability in torso but not hand motion. This indicates that, even though the participants might have produced a greater amount of torso movement in the more difficult task conditions, they did so in a manner that allowed for the coordination of their hands and torsos to increase. This pattern of results suggests that joint task constraints not only impact the relation between
Fig. 2 Mean \%Recurrence values for interpersonal handhand (right panel) and torsotorso (middle panel) coordination, and for intrapersonal hand-torso (left panel) coordination in Experiment 1
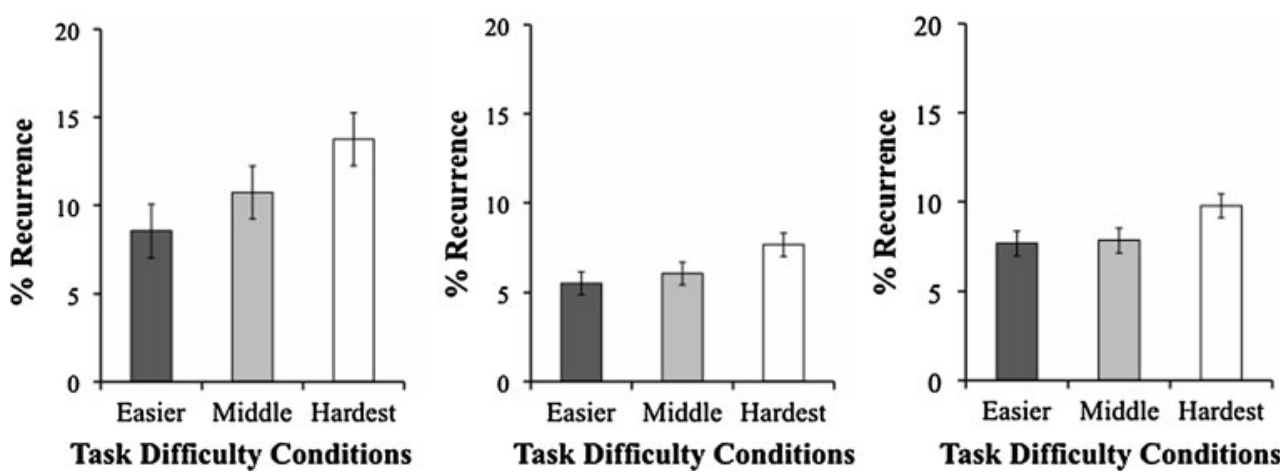
actors but also impact the way in which each actor coordinates the relative movements of his or her own body segments. Presumably, however, these changes at the intrapersonal scale were adaptive responses that facilitated the joint behavioral goal. In light of the nested nature of intrapersonal and interpersonal coordination, it seems unlikely that changes in joint task constraints could affect the two scales independently, so it is unlikely that this pattern of results means that intrapersonal and interpersonal effects merely parallel one another.

Instead, coordination at one scale appears to have had a directional influence on the other. The results present two possibilities with regard to which direction. Intrapersonal coordination effects could have been a consequence of entrainment at the interpersonal scale-as each person's hand and torso movements became more constrained by the respective segmental motions of the other person, this resulted in a tendency for their own hand and torso movements to synchronize. In this scenario, the intrapersonal coordination effects could be considered more or less epiphenomenal. Alternatively, this pattern of results could reflect the influence of intrapersonal coordination on interpersonal coordination - as each person's hand and torso movements became more tightly coupled, and interpersonal coordination of hands and torsos was facilitated.

To further investigate these possibilities, in Experiment 2 , we added a manipulation that targeted coordination at the intrapersonal scale and determined whether this led to changes in how actors coordinate with one another. We employed a tandem Romberg stance condition that reduced postural stability in the ML direction. This was expected to reduce intrapersonal hand-torso coordination (lower $\%$ Recurrence), either because it would introduce noise (increased postural sway, i.e. torso motion, would make hand-torso coordination more difficult) or because participants would have to decouple hand from torso movements in order to preserve the spatial position of the hand while the torso's motion increased.

\section{Experiment 2}

Experiment 2 aimed at determining the impact of individual constraints on interpersonal coordination measures. Two modifications to the task used in Experiment 1 were introduced. First, only the easiest and hardest task difficulty conditions were employed. Second, the balance demands of the task were varied by asking either one or the two members of the pair to perform the task in a comfortable (open-feet; same as Experiment 1) or a less familiar and putatively more difficult (tandem Romberg) postural stance. Both of these are difficulty manipulations, but not of the same nature, and thus they were not necessarily expected to have the same directional effect on intrapersonal and/or interpersonal coordination (e.g., increase the level of coordination as in Experiment 1). Decreasing the target size is an increase in task difficulty only at the scale at which the joint task is defined - the interpersonal scalebecause the size of the circle has no direct effect on an individual's ability to stand and hold the object, per se. The tandem Romberg stance increases the motor difficulty of the task for the individual by challenging balance control, because this stance reduces the ML base of support and typically results in increased postural sway variability in the ML plane (Gatev et al. 1999). The stance manipulation also increases joint task difficulty because it alters the postural backdrop against which the manual task must be performed.

Increasing the task difficulty by varying individual task demands could trickle up as noise or as a series of perturbations to hand and torso movements (indexed by increments in motion variability) that would lead to a reduction in the degree of interpersonal hand and torso coordination (indexed by \%Recurrence) (cf., Stoffregen et al. 2009). However, alternative scenarios are also possible. Compensatory changes to sustain interpersonal coordination could be expected, if the postural instability is countered by an adaptive modulation of coupling strength at either the intrapersonal or the interpersonal scale (indexed by MaxLine). Joint task performance could, as a result, be preserved or even strengthened. At the intrapersonal scale, for instance, strong synergistic coupling between an individual's hand and torso motions would allow compensation for postural instability and, therefore, preservation of a sufficient degree of interpersonal handhand coordination. At the interpersonal level, in turn, an increment in torso-torso coordination could serve to reduce incongruent postural sway between the participants and therefore make it easier to perform the joint precision task. Either outcome resulting from the postural stability manipulation would provide important clues as to the organization of joint supra-postural task performance and the relation of intrapersonal and interpersonal synergies.

\section{Methods}

\section{Participants}

Twenty-four participants (19 women, 5 men, mean age $=19.83$ years) from the University of Cincinnati participated in this study. Pairs of participants were assembled based on their height. Members of a pair differed in height by $0-12.5 \mathrm{~cm} \quad($ mean $=3.58 \mathrm{~cm} ; \mathrm{SD}=3.52)$, and in shoulder-to-fingertip length by $0.5-8.5 \mathrm{~cm} \quad$ (mean = $3.37 \mathrm{~cm} ; \mathrm{SD}=2.36 \mathrm{~cm}$ ). All participants were right-handed and had normal or corrected-to-normal vision. 


\section{Materials and apparatus}

The same materials and apparatus as Experiment 1 were employed except only the largest and smallest target circles were used.

\section{Procedure}

Procedures were identical to those employed in Experiment 1, with only one difference: Participants performed the task in either an open stance (shoulder width) or a tandem Romberg stance (right foot in front of left with left toe touching the right heel). There were three possible stance configurations: (1) both participants stood with in the tandem Romberg stance (tandem-tandem), (2) both participants stood with their feet shoulder width apart (openopen), or (3) one of the participants stood with his/her feet shoulder width apart while the other stood with his/her feet in tandem (open-tandem). Each participant completed a total of 12 trials (six trials holding the pointer and six trials holding the circle) with one trial per (difficulty $\times$ stance) condition. Each trial lasted $50 \mathrm{~s}$.

\section{Data reduction and analysis}

Time series of ML hand and torso motion on each trial for each participant were submitted to data reduction to quantify motion variability (SD) and to CRQA to quantify the same parameters (\%Recurrence and MaxLine) as in Experiment 1. Measures of hand-hand and torso-torso (interpersonal) coordination were analyzed using two-factor ANOVA with task difficulty (easiest and hardest) and stance (open-open, tandem-tandem, and open-tandem) as factors. Measures of hand and torso variability (SD), and hand-torso (intrapersonal) coordination were analyzed using two-factor ANOVA with task difficulty (easiest and hardest) and stance (open and tandem) as factors. Simpleeffects analyses and Bonferroni-Dunn post hoc tests were used as appropriate. Non-significant results are not reported.

\section{Results and discussion}

Overall, there were more performance errors than in Experiment 1, as 7 of the 12 pairs had to repeat at least one trial due to an inability to comply with the task constraints (pairs 5 and 8 had to repeat two trials). In all, two trials were repeated for the easy open-tandem stance condition, four for the hard open-tandem stance condition, and three for the hard tandem-tandem condition.

As predicted, increases in individual (stance) demands impacted the movements of the hand and torso. Hand and torso movement variability measures were larger in the tandem stance compared with the shoulder-width stance, $F(1,23)=8.20, P<.05, \eta_{p}^{2}=0.26$ and $F(1,23)=22.05$, $P<.05, \eta_{p}^{2}=0.49$, respectively. Conversely, the joint (target size) manipulation affected only hand motion, which was more variable in the more difficult small target condition than the large target condition, $F(1,23)=11.13$, $P<.05, \eta_{p}^{2}=0.33$.

The amount of interpersonal and intrapersonal coordination as indexed by \%Recurrence also varied in response to both the joint and the individual task manipulations. Decreases in target size led to increased interpersonal coordination across hands $[F(2,22)=8.26, \quad P<.05$, $\left.\eta_{p}^{2}=0.80\right]$ (easy: $m=8.9, \mathrm{SD}=1.7$; hard: $m=12.9$, $\mathrm{SD}=2$ ) (see Fig. 3, left panel), but not across torsos (easy: $m=5.8, \mathrm{SD}=1.7$; hard: $m=6.3, \mathrm{SD}=1.5$ ) (see Fig. 3, middle panel). Changes in stance affected the amount of interpersonal coordination across hands and across torsos $\left[F(1,11)=8.26, P<.05, \eta_{p}^{2}=0.35\right.$, and $F(2,22)=10.91, P<.05, \eta_{p}^{2}=0.50$, respectively]; for both segments, interpersonal coordination increased when both participants assumed the shoulder-width compared with the tandem Romberg stance (hand-hand coordination: tandem-tandem: $m=8.3, \mathrm{SD}=2.3 ;$ tandem-shoulderwidth: $m=12.6, \quad \mathrm{SD}=3.4 ;$ shoulder-width-shoulderwidth: $m=13.3, \quad \mathrm{SD}=3.9$; torso-torso coordination: tandem-tandem: $m=4.8, \mathrm{SD}=0.8 ;$ tandem-shoulderwidth: $\quad m=6.8, \quad \mathrm{SD}=1.9 ; \quad$ shoulder-width-shoulderwidth: $m=8.7, \mathrm{SD}=3.8$ ). The condition where each participant assumed a stance different from that of the other did not differ from either of the conditions in which both participants assumed the same stance.

The amount of intrapersonal coordination between hands and torsos (indexed by \%Recurrence) was similarly affected by decreases in target size $[F(1,23)=10.37$, $\left.P<.05, \quad \eta_{p}^{2}=0.31\right]$ (easy: $m=6.5, \quad \mathrm{SD}=1.6$; hard: $m=7.7, \mathrm{SD}=1.8$ ) and increases in stance difficulty $\left[F(1,23)=19.31, P<.05, \eta_{p}^{2}=0.46\right]$ (tandem: $m=8.2$, $\mathrm{SD}=2.4$; shoulder-width: $m=6, \mathrm{SD}=1.2$ ). However, the effects of the two manipulations were not independent, as there was a significant task difficulty $\times$ stance interaction, $F(1,23)=5.43, \quad P<.05, \quad \eta_{p}^{2}=0.47$. Hand-torso coordination increased for the hard compared with the easy difficulty condition for the shoulder-width stance, $t(23)=-3.28, P<.05$, but not for the tandem stance $(P>.05)$ (see Fig. 3, right panel).

For MaxLine, which indexes coupling strength, the task difficulty and stance manipulations impacted interpersonal coupling relations across hands and torsos differently. Hand-hand coordination was affected by changes in target size $\left[F(2,22)=62.85, P<.05, \eta_{p}^{2}=0.79\right]$; coupling was stronger for the hard compared with the easy condition (see 
Fig. 3 Mean \% Recurrence values for interpersonal handhand (left panel) and torso-torso (middle panel) coordination, and for intrapersonal hand-torso (right panel) coordination in Experiment 2

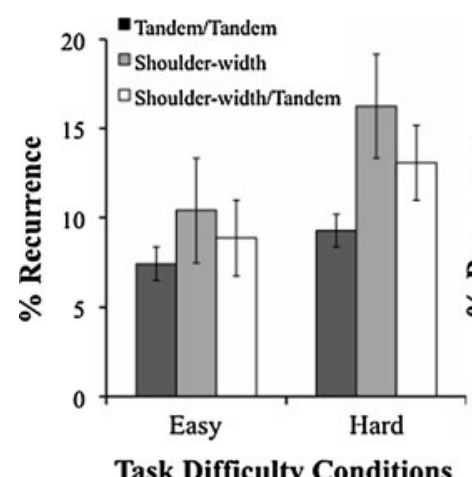

Task Difficulty Conditions

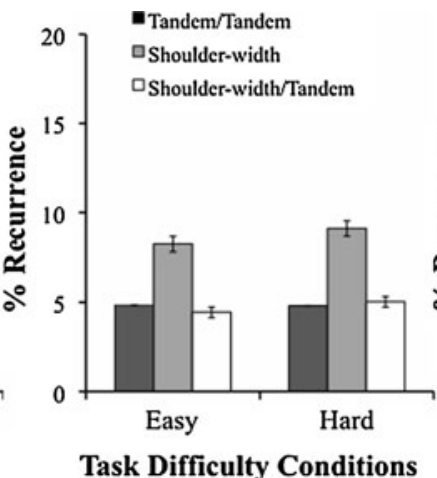

Task Difficulty Conditions

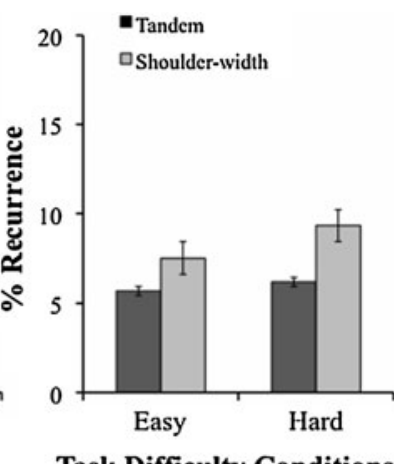

Task Difficulty Conditions
Fig. 4 Mean MaxLine values for interpersonal hand-hand (left panel) and torso-torso (right panel) coordination in Experiment 2
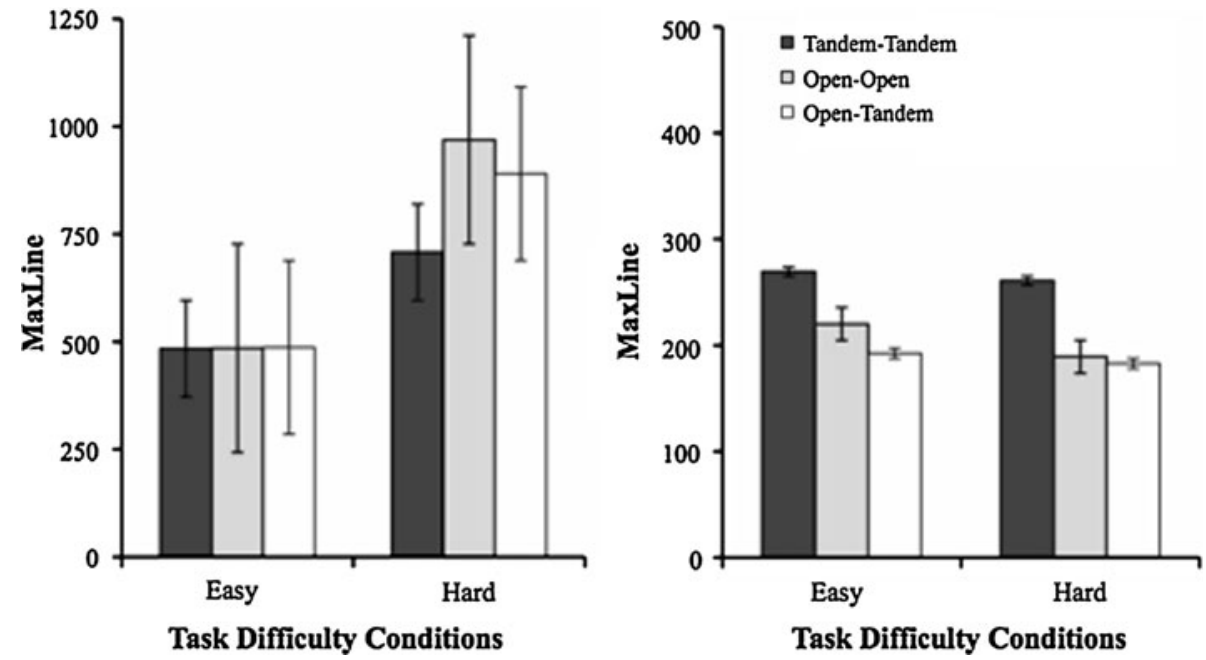

Fig. 4, left) (easy: $m=485.2, \quad \mathrm{SD}=97.8$; $\quad$ hard: $m=855.6, \mathrm{SD}=117.5)$. Conversely, torso-torso coordination was affected by the stance manipulation $[F(2,22)=$ 5.15, $\left.P<.05, \eta_{p}^{2}=0.32\right]$; coupling was stronger for the tandem/tandem compared with the shoulder-width/ shoulder-width and the tandem/shoulder-width conditions (tandem/tandem: $m=265.1, \mathrm{SD}=26.5$; tandem/shoulder-width: $\quad m=190.8, \quad \mathrm{SD}=92.9$; $\quad$ shoulder-width/ shoulder-width: $m=204.7, \mathrm{SD}=104.3$ ) (see Fig. 4, right panel). There were no MaxLine effects for intrapersonal coordination.

The results are largely consistent with those obtained in Experiment 1. The amount of interpersonal coordination again increased for the more difficult joint task condition, though the effect was in this experiment limited to handhand coordination whereas in Experiment 1 the effect was also observed for torso-torso coordination. Intrapersonal coordination was similarly affected by changes in joint task constraints but only when stance conditions were the same as in Experiment 1 (shoulder-width stance). Intrapersonal coordination between hands and torsos did not change in response to joint task constraints when the actor was standing in the more difficult tandem Romberg stance. These findings suggest that increasing the amount of motor variability at the individual level (as evidenced by increased hand and torso movement variability) might have compromised the perceptual-motor system's ability to cope with jointly imposed constraints.

Decrements in interpersonal coordination across hands and across torsos when both participants assumed a tandem Romberg stance also suggest that changes at the individual scale had an impact on the actors' ability to coordinate with one another to comply with task demands. However, interpersonal coordination did not break down completely; participants seem to have adopted a different strategy when task demands were more challenging. Increases in postural demand (task difficulty at the individual scale) were associated with an increase in interpersonal torso-torso coupling strength.

\section{General discussion}

During the performance of joint tasks, interpersonal coordination emerges spontaneously and against a background of coordinated activity within each individual (Shockley et al. 2003, 2007). Coordination between individuals and within individuals can be affected by changes in task 
demands (Stoffregen et al. 2009). In this study, we explored the relations between coordinative structures at the individual and joint scales by manipulating joint (Experiments 1 and 2) and individual (Experiment 2) task constraints. We employed a supra-postural task that required participants to hold a steady posture while jointly performing a precision motor task (keeping a stick within a circle without touching its borders). The results showed that adaptive changes occur at both the intrapersonal and the interpersonal scales in response to manipulations at either scale. This was hypothesized because coordinative processes are nested within one another during dyadic performance of tasks such as this, so interpersonal and intrapersonal coordinative processes are instantiated in overlapping structures of the same systems. The type of adaptive change that was observed, however, depended critically on the level targeted by our manipulations, revealing multiple mechanisms and processes available for enabling a dyad achieve the joint goal.

In Experiment 1, the amount of both interpersonal and intrapersonal coordination increased as the joint task precision demands (target size) were increased. Also, the amount of intrapersonal coordination between the hand and the torso was also increased in order to meet the precision demands of the task. In general, this pattern of results provides additional evidence in support of the hypothesis that interpersonal and intrapersonal coordination are governed by the same basic principles (Black et al. 2007; Schmidt et al. 1990, 1998; Schmidt and O'brien 1997; Schmidt and Turvey 1994). In Experiment 1, changes in joint task difficulty influenced the amount of interpersonal and intrapersonal coordination but not the strength of the coupling relations (MaxLine), which suggests a particular mechanism for increasing interpersonal coordination in terms of reducing fluctuations due to noise (Richardson et al. 2007b).

In Experiment 2, we added a manipulation of task difficulty at the individual level that was expected to influence interpersonal coordination as a source of unwanted postural variability. When participants performed the supra-postural task in the less stable tandem Romberg posture, the amount of motor variability coordinative structures had to absorb in order to perform the task successfully increased, and interpersonal coordination across hands and across torsos decreased. Those \% Recurrence results are in line with the results, showing that interpersonal coordination observed during conversation was attenuated when participants both stood on an unstable surface that increased balance demands (Stoffregen et al. 2009).

Independent of the stance condition, in Experiment 2, increased joint task difficulty was associated with a greater degree of interpersonal coordination of hand movements. Whereas in Experiment 1 and in the shoulder-width stance condition in Experiment 2 this result was accompanied by an increase in intrapersonal hand-torso coordination, in the tandem Romberg stance participants were apparently unable to increase intrapersonal hand-torso coordination as the joint task became more difficult. Coupling hand and torso movement could facilitate performance of difficult precision tasks, for example, by countering an unwanted or unexpected fluctuation in torso position by an adaptive, compensatory change in hand position. However, this strategy may not be possible in the tandem Romberg stance condition where the need to resolve postural instability might be paramount (Mitra and Fraizer 2004). These findings suggest there are multiple, adaptive mechanisms for achieving or preserving sufficiently high levels of interpersonal hand coordination to enable achievement of a joint goal. Some of these mechanisms appear to engage intrapersonal coordination patterns that directly promote interpersonal coordination when joint task difficulty increases, whereas other mechanisms permit interpersonal coordination to increase when joint task difficulty increases in the absence of a parallel change in intrapersonal coordination. When the ability to adaptively increase intrapersonal hand-torso coordination was diminished in the tandem Romberg stance, a compensatory increase in torsotorso (interpersonal) coupling strength may have provided the necessary degree of coordinative stability to achieve the joint goal.

Implications for understanding other types of joint action

Overall, these findings add to a growing body of literature that highlights the importance of coordinative processes in everyday interactions. They also provide further evidence of the sophistication and flexibility of our perceptual-motor systems and their ability to adapt to changing demands while supporting joint actions. In that respect, these findings provide some important insights into the relation between intrapersonal and interpersonal coordination. They indicate that constraints operating at the individual and joint scales have reciprocal effects than span scales: intrapersonal constraints affect processes of both intrapersonal and interpersonal coordination, and likewise interpersonal constraints affect processes of both intrapersonal and interpersonal coordination. More importantly, they suggest that adjustments originating at the intrapersonal level help support and maintain coordination at the interpersonal level.

These findings are consistent with others, suggesting that the coordination of attention, or the establishment of joint attention to the same task and environmental stimuli, facilitates joint task performance. During joint tasks, when two or more people interact to accomplish a shared goal, 
actors coordinate their cognitive processes along with their motor outputs as the action unfolds in real time in order to achieve a shared goal (Clark 1996). Richardson and Dale (2005), and Richardson et al. (2007b) have suggested that increased spatial-temporal coordination of motor behavior (e.g., eye movements, postural sway, or limb movements) is a strong indicant of the coordination of attention between individuals (Richardson et al. 2008; Shockley et al. 2003). In this light, increases in both intrapersonal and interpersonal coordination may represent increased attention devoted to the sub-tasks and a coordination of attention across individuals.

This has an important implication for instances where the outcome variable of interest is joint attention, rather than perceptual-motor performance. If interpersonal perceptual-motor coordination embodies joint attention and the coordination of cognitive activity involved in joint tasks, then perceptual-motor processes may provide a foothold for understanding the fundamental constraints that also characterize cognitive coordination during joint action. The existence of well-developed frameworks for measuring, analyzing, and modeling motor coordination dynamics could inform understanding of cognitive coordination and help bridge the gaps between models of motor coordination and models of cognitive processes (Shockley et al. 2009).

\section{References}

Amazeen PG, Schmidt RC, Turvey MT (1995) Frequency detuning of the phase entrainment dynamics of visually coupled rhythmic movements. Biol Cybern 72(6):511-518

Balasubramaniam R, Riley MA, Turvey MT (2000) Specificity of postural sway to the demands of a precision task. Gait Posture 11(1):12-24

Bernstein N (1967) Co-ordination and regulation of movements. Lancet 1(7500)

Black DR, Riley MA, McCord CK (2007) Synergies in intra-and interpersonal interlimb rhythmic coordination. Mot Control 11(4):348-373

Clark HH (1996) Using language. Cambridge University Press, Cambridge

Fowler CA, Richardson MJ, Marsh KL, Shockley KD (2008) Language use, coordination, and the emergence of cooperative action. Coordination: Neural, behavioral and social dynamics, pp 261-279-354

Gatev P, Thomas S, Kepple T, Hallett M (1999) Feedforward ankle strategy of balance during quiet stance in adults. J PhysiolLond 514(3):915-928

Gelfand IM, Tsetlin ML (1966) On mathematical modeling of the mechanisms of the central nervous system. In: Gelfand IM, Gurfinkel VS, Fomin SV, Tsetlin ML (eds) Models of the structural-functional organization of certain biological systems. MIT Press, Cambridge, pp 9-26

Knoblich G, Sebanz N (2006) The social nature of perception and action. Curr Dir Psychol Sci 15(3):99-104
Latash ML (2008) Neurophysiological basis of movement, 2nd edn. Human Kinetics, Urbana

Marsh KL, Richardson MJ, Baron RM, Schmidt RC (2006) Contrasting approaches to perceiving and acting with others. Ecol Psychol 18(1):1-38

Marsh KL, Richardson MJ, Schmidt RC (2009) Social connection through joint action and interpersonal coordination. Top Cogn Sci 1:320-339

Mitra S, Fraizer EV (2004) Effects of explicit sway-minimization on postural-suprapostural dual-task performance. Hum Mov Sci 23(1):1-20. doi:10.1016/j.humov.2004.03.003.pii:S016794570 4000193

Oullier O, Deguzman G, Jantzen KJ, Lagarde J, Kelso JAS (2008) Social coordination dynamics: visual information exchange mediates spontaneous phase synchrony between people. Soc Neurosci 3:178-192

Pellecchia GL, Shockley K, Turvey MT (2005) Concurrent cognitive task modulates coordination dynamics. Cogn Sci 29(4):531-557

Ramenzoni VC, Riley MA, Shockley K, Baker AA (under review) Interpersonal and intrapersonal coordinative modes for joint and single task performance

Riccio GE, Stoffregen TA (1988) Affordances as constraints on the control of stance. Hum Mov Sci 7(2-4):265-300

Richardson D, Dale R (2005) Looking to understand: the coupling between speakers' and listeners' eye movements and its relationship to discourse comprehension. Cogn Sci (29):10451060

Richardson DC, Dale R, Kirkham NZ (2007a) The art of conversation is coordination-common ground and the coupling of eye movements during dialogue. Psychol Sci 18(5):407-413

Richardson MJ, Schmidt RC, Kay BA (2007b) Distinguishing the noise and attractor strength of coordinated limb movements using recurrence analysis. Biol Cybern 96(1):59-78. doi: 10.1007/S00422-006-0104-6

Richardson D, Dale R, Shockley K (2008) Synchrony and swing in conversation: coordination, temporal dynamics and communication. Embodied communication. Oxford University Press, Oxford

Richardson MJ, Marsh KL, Schmidt RC (2010) Challenging egocentric notions of perceiving, acting and knowing. The mind in context. Guilford, New York

Riley MA, Stoffregen TA, Grocki MJ, Turvey MT (1999) Postural stabilization for the control of touching. Hum Mov Sci 18(6):795-817

Riley MA, Richardson MJ, Shockley K, Ramenzoni VC (2011) Interpersonal synergies. Front Psychol 2:38

Schmidt RC, O'brien B (1997) Evaluating the dynamics of unintended interpersonal coordination. Ecol Psychol 9(3):189-206

Schmidt RC, Richardson MJ (2008) Dynamics of interpersonal coordination. In: Jirsa AFV (ed) Coordination: neural, behavioral and social dynamics. Springer, Germany, pp 281-308

Schmidt RC, Turvey MT (1994) Phase-entrainment dynamics of visually coupled rhythmic movements. Biol Cybern 70(4):369-376

Schmidt RC, Carello C, Turvey MT (1990) Phase-transitions and critical fluctuations in the visual coordination of rhythmic movements between people. J Exp Psychol-Hum Percept Perform 16(2):227-247

Schmidt RC, Bienvenu M, Fitzpatrick P, Amazeen P (1998) A comparison of within- and between-person coordination: coordination breakdown and coupling strength. J Exp Psychol: Hum Percept Perform (24):884-900

Sebanz N, Bekkering H, Knoblich G (2006) Joint action: bodies and minds moving together. Trends Cogn Sci 10(2):70-76. doi: 10.1016/J.Tics.2005.12.009

Shockley K (2005) Cross recurrence quantification of interpersonal postural activity. In: Orden MARGCV (ed) Tutorials in 
contemporary nonlinear methods for the behavioral sciences, pp 142-177. http://www.nsf.gov/sbe/bcs/pac/nmbs/nmbs.jsp. Retrieved 5 May 2005

Shockley K, Turvey MT (2005) Encoding and retrieval during bimanual rhythmic coordination. J Exp Psychol-Learn Mem Cogn 31(5):980-990. doi:10.1037/0278-7393.31.5.980

Shockley K, Turvey MT (2006) Dual-task influences on strategic retrieval and coordination dynamics. Psychon Bull Rev (13):985-990

Shockley K, Butwill M, Zbilut JP, Webber CL (2002) Cross recurrence quantification of coupled oscillators. Phys Lett A 305(1-2):59-69 (pii: S0375-9601(02)01411-1)

Shockley K, Santana MV, Fowler CA (2003) Mutual interpersonal postural constraints are involved in cooperative conversation. J Exp Psychol-Hum Percept Perform 29(2):326-332. doi: 10.1037/0096-1523.29.2.326

Shockley K, Baker AA, Richardson MJ, Fowler CA (2007) Articulatory constraints on interpersonal postural coordination. J Exp Psychol-Hum Percept Perform 33(1):201-208. doi: 10.1037/0096-1523.33.1.201

Shockley K, Richardson DC, Dale R (2009) Conversation and coordinative structures. Top Cogn Sci 1(2):305-319

Stoffregen TA, Smart LJ, Bardy BG, Pagulayan RJ (1999) Postural stabilization of looking. J Exp Psychol-Hum Percept Perform 25(6): 1641-1658

Stoffregen TA, Pagulayan RJ, Bardy BG, Hettinger LJ (2000) Modulating postural control to facilitate visual performance. Hum Mov Sci 19(2):203-220
Stoffregen TA, Giveans MR, Villard S, Yank JR, Shockley K (2009) Interpersonal postural coordination on rigid and non-rigid surfaces. Mot Control 13(4):471-483

Temprado JJ, Swinnen SP, Carson RG, Tourment A, Laurent M (2003) Interaction of directional, neuromuscular and egocentric constraints on the stability of preferred bimanual coordination patterns. Hum Mov Sci 22(3):339-363. doi:10.1016/S01679457(03)00049-6

Turvey MT (1977) Contrasting orientations to theory of visual information-processing. Psychol Rev 84(1):67-88

Turvey MT (1990) Coordination. Am Psychol 45(8):938-953

Turvey MT, Shaw RE, Mace W (1978) Issues in the theory of action: degrees of freedom, coordinative structures and coalitions. In: Requin J (ed) Attention and performance VII. Lawrence Erlbaum Associates, Hillsdale, pp 567-595

Whiting HTA, Vogt S, Vereijken B (1992) Human skill and motor control: some aspects of the motor control-motor learning relation. In: Summers JJ (ed) Approaches to the study of motor control and learning. North Holland, Amsterdam, pp 81-111

Wilson M, Knoblich G (2005) The case for motor involvement in perceiving co-specifics. Psychol Bull (131):460-473

Zbilut JP, Webber CL (1992) Embeddings and delays as derived from quantification of recurrence plots. Phys Lett A 171(3-4):199 203

Zbilut JP, Giuliani A, Webber CL (1998) Detecting deterministic signals in exceptionally noisy environments using cross-recurrence quantification. Phys Lett A 246(1-2):122-128 